\title{
A huge nasal extramedullary plasmacytoma: Computerize tomography and diffusion magnetic resonance imaging findings
}

\author{
Muzaffer Elmalıa, Ahmet Veysel Polat ${ }^{a}$, Ramazan Aydın*, Yurdanur Süllü ${ }^{\mathrm{b}}$, Mehmet Koyuncu \\ ${ }^{a}$ Department of Radiology, Faculty of Medicine, Ondokuz Mayis University, Samsun,Turkey \\ ${ }^{b}$ Department of Pathology, Faculty of Medicine, Ondokuz Mayis University, Samsun, Turkey \\ ${ }^{c}$ Department of Otorhinolaryngology, Faculty of Medicine, Ondokuz Mayis University, Samsun,Turkey
}

\section{ARTICLE INFO}

\section{Article History}

Received

$11 / 06 / 2012$

Accepted

$11 / 06 / 2012$

\section{* Correspondence to:}

Ramazan Aydın

Ondokuz Mayıs University.

Faculty of Medicine,

Department of Radiology,

Samsun, Turkey

e-posta: raydin1984@hotmail.com

\section{ABSTRACT}

Extramedullary plasmacytoma (EMP) is an uncommon malignant neoplasm arising outside the bone marrow without clinical evidence of multiple myeloma. EMPs represent less than $1 \%$ of all head and neck malignancies. A 51-year-old male had a history of nasal obstruction and epistaxis for several months. Physical examination revealed a fragile and hemorrhagic mass in the nasal cavity. CT scan showed a huge soft tissue mass in the nasal cavity. On the MR images, there was a soft tissue mass which showed marked enhancement after gadolinium administration and restricted diffusion on diffusion weighted images. Histopathological examination results revealed atypical plasma cell infiltration. A diagnosis of EMP was made with these findings. The data of imaging features of nasal EMP is very few in the literature. Therefore, we aimed to present CT and MRI findings of a patient with EMP and contribution of diffusion MRI to the diagnosis.

J. Exp. Clin. Med., 2013; 30:81-83

\section{Keywords:}

Computerized tomography

Diffusion magnetic resonance imaging

Extramedullary plasmacytoma

Magnetic resonance imaging

Plasmacytoma

(C) 2013 OMU

\section{Introduction}

Extramedullary plasmacytoma (EMP) is an uncommon malignant neoplasm arising outside the bone marrow without clinical evidence of multiple myeloma. EMP, which can occur in virtually any part of the body, shows a predilection for the head and neck region (Hidaka et al., 2000). EMPs represent less than $1 \%$ of all head and neck malignancies (Maniglia and Xue, 1983). The data of imaging features of nasal EMP is very few in the literature. Therefore, we aimed to present CT and MRI findings in a patient with the diagnosis of nasal EMP.

\section{Case}

A 51-year-old male had a history of nasal obstruction and epistaxis for several months. The patient had no previous history of any disease. Physical examination revealed a fragile and hemorrhagic mass in the nasal cavity. CT scan showed a soft tissue mass that was $4 \times 5 \mathrm{~cm}$ in size. There was nasal septal deviation to the right side and compressed left maxiller sinus caused by the mass (Fig. 1). On the MR images, there was a soft tissue mass which was slightly hyperintense on T1- weighted images and heterogenous hyperintense on T2weighted images compared to the muscle.

The mass showed marked enhancement after gadolinium administration. The mass is seen hyperintense on diffusion weighted images (DWI) and hypointense on apperent diffusion coefficient (ADC) maps. Median ADC value of the mass was measured as $0.9 \times 10^{-3} \mathrm{~mm}^{2} / \mathrm{sn}$ (restricted diffusion) (Fig. 2).

Histopathological examination results revealed atypical plasma cell infiltration. These atypical plasma cells were stained positive stained with CD138 and Kappa, negative with Lambda (Fig. 3). Skeletal survey, blood and urine tests were within normal limits. A diagnosis of EMP was made with these findings. 

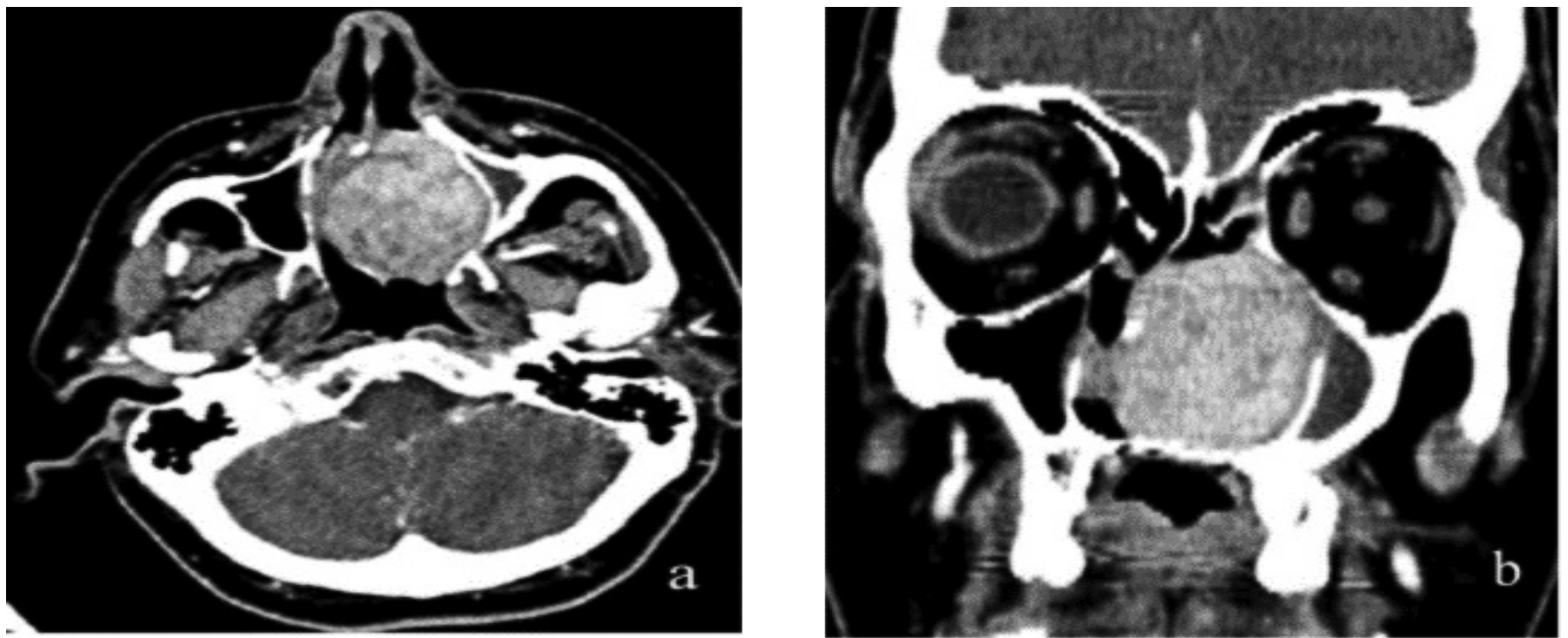

Fig. 1. Axial (a) and coronal (b) CT images show a markedly enhancing huge soft tissue mass in the left nasal cavity.

\section{Discussion}

EMP of the nasal tract is a rare neoplasm of B-lymphocyte populations. They account for less than $1 \%$ of all head and neck malignancies and $4 \%$ of non-epithelial tumors of the na- sal tract with an overall incidence of $3 / 100.000$ population per year (Rodriguez-de-Velasquez et al., 1996). The incidence is higher in men aged between $60-80$ years, with a male-to-female ratio of more than 3:1. EMP appears as a slowly growing
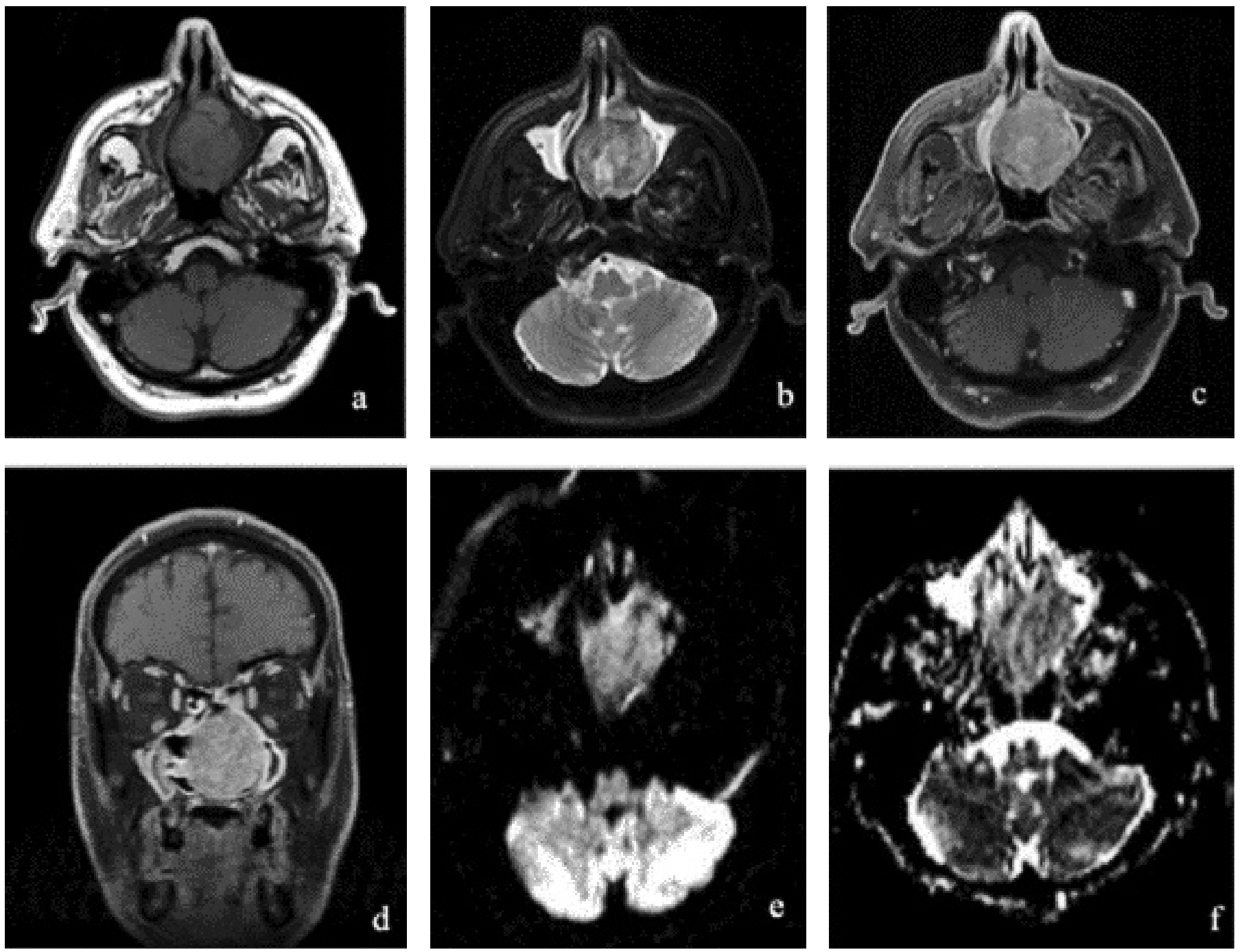

Fig. 2. MR images show a soft tissue mass that was slightly hyperintense on T1- weighted axial image (a), heterogenous hyperintense on T2weighted axial image (b) compared to the muscle. The mass showed markedly enhancement after gadolinium administration on fat- suppressed axial T1 (c) and coronal T1- weighted (d) images. The mass is seen hyperintense on DWI images (e) and hypointense on ADC maps (f). 


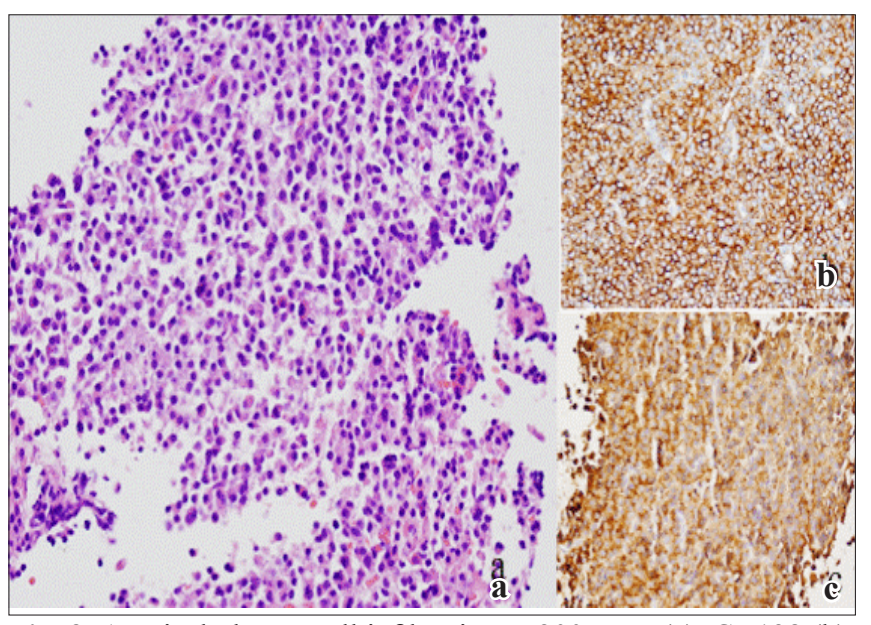

Fig. 3. Atypical plasma cell infiltration, X200; H-E (a), CD138 (b), Kappa (c).

mass, therefore, a delay in diagnosis is common. The clinical presentation is mainly due to mass effect and depends on the site of involvement (Ching et al., 2002). Plasmacytoma of the head and neck is often bulky, and is characterized by a combination of expansion with remodelling of bone and lytic bone destruction. CT and MRI are the most preferable modalities in both diagnosis and treatment of EMP. The effect of mass on the bone is best evaluated by CT. MRI is superior when it comes to showing the internal structure of the mass, presence of enhancement and invasion to the surrounding tissues. DWI can visualize the random thermal motion of molecules known as Brownian motion, causing incoherent phase shifts resulting in signal attenuation. Thus, this technique allows for quantification of diffusion by calculating the ADC.

In malignant tumors the increased cellular density restricts water diffusion in the interstitial space, resulting in lower ADC values (Matoba et al., 2007). There are a few reports about EMP in the literature but we found only two literature concerning MRI features of nasal EMP. Besides we did not find any case of DWI concerning EMP but however, we came across with one case of plasmacytoma of the sternum that DWI was performed (Nakanishi et al., 2010). The MRI study reveals low to intermediate signal intensity on T1- and moderate to marked increased signal intensity on T2-weighted images. Following the administration of gadolinium, there is moderate to marked enhancement. The mass is seen hyperintense on DWI, hypointense on ADC maps (restricted diffusion). Imaging alone cannot be used to reliably distinguish this tumor from other malignancies of the nasal tract such as squamous cell carcinoma, lymphoma, adenocystic carcinoma, esthesioneuroblastoma, rhabdomyosarcoma, and Wegener granulomatosis. Together, CT and MRI are the methods of choice for assessing local disease, but the diagnosis of EMP of the nasal tract still requires histologic and immunohistochemical evidence. This tumor is highly radiosensitive. Some clinicians favor radiotheraphy and others favor surgical management. Our patient was planned to take radiotherapy. Before making the diagnosis of EMP, it is important to differentiate whether it is a plasma cell dyscrasia. Because, EMP and solitary plasmacytoma of the bone have better prognosis compared with multiple myeloma. Local recurrence may occur in 6 to 10 percent of cases that have had adequate initial treatment (Wax et al., 1993). Furthermore, progression to multiple myeloma has been reported to occur in 10 to 30 percent of EMP cases within the first two years (Holland et al., 1992). Therefore, long-term follow-up including not only a local control but also systemic observations via blood counts or immunoglobulin measurement are necessary.

\section{REFERENCES}

Ching, A.S., Khoo, J.B., Chong, V.F., 2002. CT and MR imaging of solitary extramedullary plasmacytoma of the nasal tract. Am. J. Neuroradiol. $23,1632-1636$.

Hidaka, H., Ikeda, K., Oshima, T., Ohtani, H., Suzuki, H., Takasaka, T., 2000. A case of extramedullary plasmacytoma arising from the nasal septum. J. Laryngol. Otol. 114, 53-55.

Holland, J., Trenkner, D.A., Wasserman, T.H., Fineberg, B., 1992. Plasmacytoma. Treatment results and conversion to myeloma. Cancer. 69, 1513-1517.

Maniglia, A.J., Xue, J.W., 1983. Plasmacytoma of the larynx. Laryngoscope. 93, 741-744.

Matoba, M., Tonami, H., Kondou, T., Yokota, H., Higashi, K., Toga, H., Sakuma, T., 2007. Lung carcinoma: Diffusion-weighted MR imagingpreliminary evaluation with apparent diffusion coefficient. Radiology. 243, 570-577.

Nakanishi, K., Kashiwagi, N., Hamada, K., Yagi, T., Tomita, Y., 2010. Solitary plasmacytoma of the sternum detected incidentally by MR imaging of the cervical spine. Magn. Reson. Med. Sci. 9, 227-231.

Rodriguez-de-Velasquez, A., Weber, A.L., Montgomery, W., 1996. Extramedullary laryngeal plasmacytoma. Ann. Otol. Rhinol. Laryn. 105, 483-486.

Wax, M.K., Yun, K.J., Omar, R.A., 1993. Extramedullary plasmacytomas of the head and neck. Otolaryng. Head. Neck. Surg. 109, $877-885$. 